Citation: Efe-Ononeme, O. E., Ikpe, A. E., Ariavie, G. O., " Modal Analysis of Conventional Gas Turbine Blade Materials (Udımet 500 and In738) for Industrial Applicatıons". Journal of Engineering Technology and Applied Sciences 3 (2) 2018 : 119-133.

\title{
MODAL ANALYSIS OF CONVENTIONAL GAS TURBINE BLADE MATERIALS (UDIMET 500 AND IN738) FOR INDUSTRIAL APPLICATIONS
}

\author{
Oghenefejiro E. Efe-Ononeme, Aniekan E. Ikpe*, Godfrey O. Ariavie \\ Room 142, Department of Mechanical Engineering, Faculty of Engineering, University of Benin, \\ Benin City, Edo State, P.M.B. 1154, Nigeria, \\ *ikpeaniekan@gmail.com (corresponding author)
}

\begin{abstract}
Finite element method (FEM) was utilized to determine the natural frequency of two turbine blade materials applicable to Trans-amadi power plant in Port Harcourt, Nigeria. Comparing the two gas turbine blade materials, the fundamental frequency under the same load condition was $751 \mathrm{~Hz}$ for IN 738 and $896 \mathrm{~Hz}$ for U500 turbine blade material. This implies that the natural frequencies obtained for both materials were much higher than the operational frequency of $85 \mathrm{~Hz}$ for resonance to occur. Therefore, resonance would be delayed across the blade materials in service condition, indicating that gas turbine blades designed with both materials would be dynamically stable under operational frequency approaching $745 \mathrm{~Hz}$. It was observed that U500 gas turbine blade material which had a higher fundamental frequency has a better mechanical properties that can undergo a longer service condition in extreme working phase before failure compared to IN 738 material. This justification was deduced from the difference between the operational frequency and the fundamental frequency; as the closer the value obtained for fundamental frequency to the operational frequency, the higher the possibility of failure and vice versa. To avoid unforeseen failure and downtime in service operation, vibration test should be conducted on routine schedule in order to meet the expected performance of the gas turbine blade.
\end{abstract}

Keywords: Gas turbine, turbine blade, failure, resonance, natural frequency, vibration.

\section{Introduction}

The gas turbine engine is a critical unit in the operation of power plants, aircraft and heavy duty vehicles. It consists of three main parts namely; compressor, combustion chamber and the turbine. Failure of a gas turbine can cause significant negative effects on power generation, as a result of poor maintenance and monitoring. Vibration analysis is an approach 
that allows early detection of faults in most rotor dynamic equipment such as motors, compressor, gears and turbine etc. The cause of vibration is as a results of resonance, rubbing parts, unbalanced rotating parts, misalignment of couplings and bearings, bent shafts, mechanical looseness etc. [1]. Vibrations in rotating machineries are unavoidable and it is expected rotating members to operate within acceptable level to prevent upsurge in vibration. When the members of a rotating body such as the gas turbine system misaligns with one another or generates excessive noise, vibration is likely to set in and if not rectified, the problem may intensify and eventually lead to failure [2,3]. Blades are the heart of a gas turbine engines and serves as the medium of transfer of energy from the gases to the turbine rotor. The conditions which these blades operate make them most susceptible to failure and demand periodic inspection. Turbo-machinery blade failures are mainly caused by high cycle fatigue which arises as a result of mechanical vibration resulting from high alternating stresses $[4,5,6]$. According to Kalapala [7], about $42 \%$ of the total gas turbine failures are caused by vibration induced fatigue. The causes of excitation are due to inlet distortion resulting in nonuniform flow fields, pressure disturbances from adjacent blade rows, and wakes. The vibration problem can be avoided by avoiding resonant conditions. Therefore, the blade must be designed in a manner that accommodate the excitation forces, in order to minimize the amplitude of vibration which could lead to blade failure. Natural frequency is the frequency at which an object vibrates when excited by a force. If the structure possesses least resistance to the force, the tendency of failure may be high if not controlled. Mode shape is the deflection of an object at a given natural frequency. If a structure vibrates at frequencies higher or closer to the natural frequency of the body, the vibration may be exceptionally high. It is therefore necessary to assess the operational natural frequencies of structures under loading [8]. The eigenvalue (natural frequency) and eigenvector (mode shape) are calculated to define the dynamics of the structure. Holographic method is employed for determining mode shapes. There are several modes which occur at various excitation frequencies such as first flexural or first flap (1F) mode, second flap mode (2F), torsional mode, axial mode and First edgewise (1E) mode of excitation. As employed in this study, modal analysis is an approach that can be used in assessing the structural behaviour of machine components which may include vibration, damping, mass, modes shapes (dynamic properties) and frequencies [9]. Generally resonant vibrations can be characterised by simple and effective use of Modes. Modes are known to be inherent properties of a structure. This within a structure is caused by interaction between the elastic properties and inertia of materials. In structural analysis, modal analysis helps to identify areas of weakness in design that needs improvements [10]. Resonance is a condition where there is maximum amplitude of vibration and minimum resistance to oscillating force. For resonance condition to occur, the shape and frequency of a force must correspond to the natural frequency and mode shape of the structure (blade). However, the use of dampers can help in avoiding or preventing failure resulting from resonance [7].

Many research have suggested various methods to describe the causes of failure in turbine blades. A lot have been documented to ensure the continuity of knowledge in failure analysis with concentration on issues arising from vibration as it affects other components of the gas turbine engine. Srinivasan [11] investigated the effects of blade thickness and blade length on the performance of a gas turbine engine and found that the blade thickness reduced as the fatigue strength also reduced which is not desirable for service operation of a turbine system. Moreover, the blade length reduced as angular speed also reduced, therefore, concluding that shorter turbine blades are ideal for turbine systems.

Adawi [12] carried out studies to analyze the problem associated with high vibrations in gas turbine installed in Al Ghubra Power and Desalination plant. Case studies related to vibration 
problems were evaluated to diagnose the cause of vibration and to find the root cause of failure for the gas turbine system. Data were analyzed for the high vibration condition and corrective action were employed to rectify the problem to reduce vibration level. Bhupendra [9] carried out modal analysis on vibration in gas turbine blades and also effects of vibration on the blade. Their investigation provided various range of frequencies in different modes, and the behavior of the blade material $(\mathrm{N}-155)$ for various ranges of frequency was used to determine possible deformations. By studying this deformations it was important to check whether or not the deformations are detrimental to blade the material and coatings.

Lee [13] proposed a technique for mounting the blade on the hub, but arc welding and forging are being used presently in the industry. Kalapala [7] conducted a research work on modal analysis of the turbine blade, using ANSYS V-14 to determine the natural frequency and critical speeds for three different blade materials (Nickel-Chromium alloy, stainless steel, and titanium alloy). When compared to other materials, they found out that Nickel-Chromium alloy produced a better result. Sushila [14] investigated the causes of gas turbine blade failure. It was found that surface degradation due to overheating, oxidation, fatigue, degradation of coatings, hot corrosion, sulphidation, embrittlement and thermal aging are the major causes.

In the analysis of gas turbine blades, discretizing a freestanding blade and using appropriate element relations is more advantageous than the continuum approach, in that, it is simpler to carry out the analytical work. Some of these techniques and methods such as Holzer Method, Myklestad/Prohl Method, Matrix Method, Finite Difference Method, and Finite Element Method (FEM) etc. are used to broadly classify the discrete analysis of turbine blades. Finite element method was employed in this study to determine the combined bending and torsional vibration modes taking into account the effects of root flexibility and natural frequency. Finite element method is very popular and advantageous for complex problems. It has been employed in this present work.

\section{Materials and Method}

Modal Analysis was carried out on IN738 and U500 nickel based alloy which are conventionally used for gas turbine blade applications. The IN738 and U500 material properties are presented in Table 1;

Table 1. Material Properties of U500 and IN738

\begin{tabular}{lll}
\hline Materials & U 500 & IN 738 \\
\hline Specific Heat $(\mathrm{J} / \mathrm{KgK})$ & 500 & 510 \\
Young's Modulus $(\mathrm{GPa})$ & $190-210$ & 149 \\
Density $\left(\mathrm{kg} / \mathrm{m}^{3}\right)$ & 7800 & 8550 \\
Poisson's ratio & $0.27-0.30$ & 0.30 \\
Thermal conductivity $(\mathrm{W} / \mathrm{mk})$ & 16.2 & 14.3 \\
Thermal expansion $\left({ }^{0} \mathrm{C}\right)$ & $17.5 \times 10^{-6}$ & $12.5 \times 10^{-6}$ \\
Yield strength $(\mathrm{MPa})$ & 275 & 792.897 \\
Melting temperature $\left({ }^{0} \mathrm{C}\right)$ & 1360 & 1400 \\
Bulk Modulus $(\mathrm{Pa})$ & $1.583 \times 10^{11}$ & $1.247 \times 10^{10}$ \\
Shear Modulus $(\mathrm{Pa})$ & $7.307 \times 10^{10}$ & $5.730 \times 10^{10}$ \\
\hline
\end{tabular}

FEA produces a much more detailed set of results than experimental investigations and is often quicker and less expensive. Finite element modal analysis employed in this study relies 
on the discretization of the geometry to solve complex structural equations by effectively subdividing the structure in an assembly of simple finite elements. The FEA configured the turbine blade model for analysis using a complex system of points or nodes connected into a grid known as mesh. The nodes were arranged at a specific density throughout the model. The steps employed in the course of the analysis are as follows;

i. Prepare a three dimensional model in SOLIDWORKS 2014.

ii. Import the SOLIDWORKS model in ANSYS 15.0 software.

iii. Mesh the ANSYS model.

iv. Apply boundary conditions and

v. Solve the system equations to find out the unknowns.

vi. Validation of solutions obtained with the operating conditions

\subsection{Operating (Boundary) Conditions}

The operating (boundary) conditions subjected to the blade as well as the required data needed for the modeling and analysis of gas turbine blade failure are gotten from this power plant.

This work is limited to various assumptions. This includes the fact that during this analysis:

i. The material is considered to be non-linear.

ii. The body is subject to steady load. The system is assumed to be not damped.

iii. The load and structural response are assumed to vary slowly with respect to time.

iv. The type of loading are externally applied force and pressure, steady-state inertial force (rotational velocity and centrifugal force), and temperature.

v. Blade has constant cross sectional area. Mechanical analysis is carried out.

\subsection{Trans-Amadi Power Plant}

The model was obtained from Trans-Amadi Power Plant, located in Port Harcourt, Rivers State Nigeria. The power plant is sited in a land area of about 4 Hectares with a total installed capacity of $136 \mathrm{MW}$. The power plant was commissioned in 2 phases. The first phase consist of a 3x12MW Solar Mars Gas Turbines which was commissioned in 2002 while the second phase consist of a 4x25MW Nuovo Pignone frame 5 Gas Turbine, commissioned in 2010. Two of the Phase 2 turbines were procured in 2004 and the other two (2) were bought in 2005. Geographical Location of Trans-Amadi Power Plant is shown in Figure 1 while Figure 2 represent the gas turbine blade geometry in SOLIDWORKS and meshed gas Turbine blade using ANSYS; 


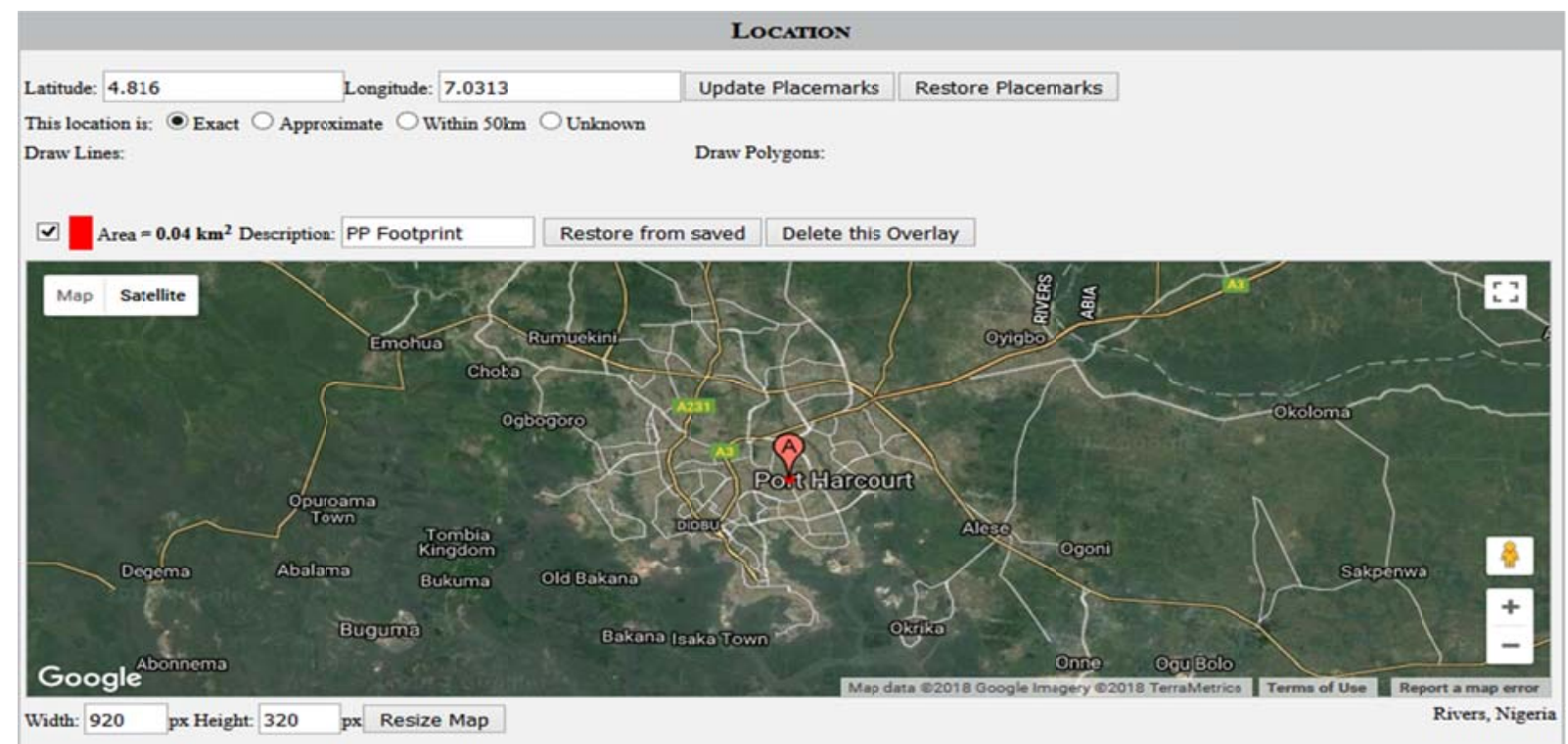

Figure 1. Geographical Location of Trans-Amadi Power Plant
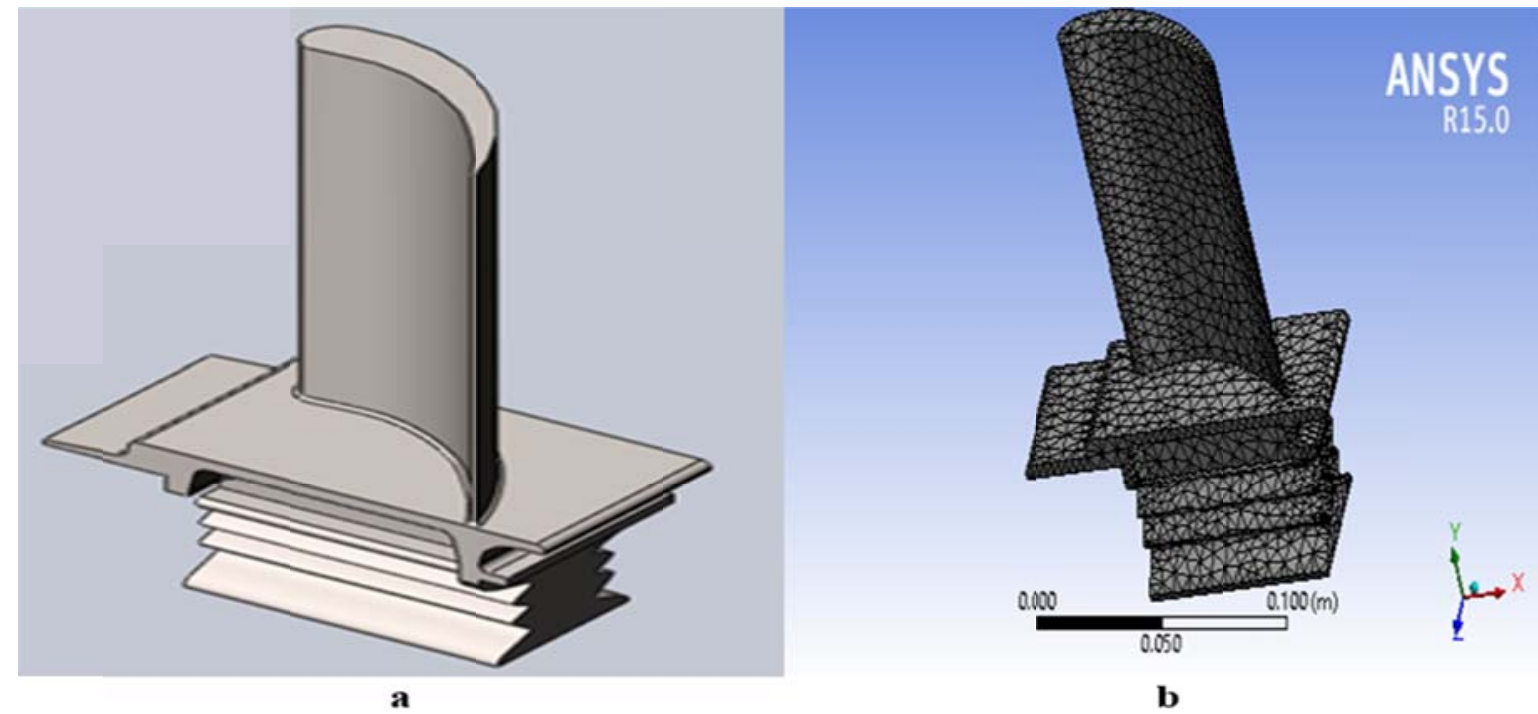

Figure 2. a. Gas Turbine Blade Geometry in SOLIDWORKS, b. Meshed Blade using ANSYS

The geometry of the gas turbine blade is modeled in SOLIDWORKS and then imported into ANSYS for meshing. The aero foil profile of the rotor blade was generated on the XZ plane with the help of key points defined by the coordinates. Then a number of splines were fitted through the key points, creating the $2 \mathrm{D}$ aero foil shape. The geometry of the blade was meshed with 8 nodded tetrahedral brick element but the element type used for this purpose was Tetrahedron 10. It has 3 degrees of freedom per node, that is, translation in $\mathrm{X}, \mathrm{Y}$ and $\mathrm{Z}$ directions were used. The element has plasticity, creep, swelling, stress stiffening, large deflection, and large strain capabilities etc. The blade dimensions are given in Table 2; 
Table 2. Gas Turbine Blade Dimensions

\begin{tabular}{ll}
\hline Parameter & Values \\
\hline Name & NACA6409 9\% \\
Blade Span (mm) & $120 \mathrm{~mm}$ \\
Blade Axial Chord Length $(\mathrm{mm})$ & $68.30 \mathrm{~mm}$ \\
Blade Root Length (mm) & $60 \mathrm{~mm}$ \\
Thickness (\%) & 100 \\
Pitch (deg) & 14 \\
\hline
\end{tabular}

The rotational speed, gas pressure and forces acting on the gas turbine blade during operation are presented in Table;

Table 3. Operational Parameters of the Gas Turbine Blade

\begin{tabular}{lc}
\hline Operating Parameters & Values \\
\hline Pressure (bar) & 8 \\
Speed (rpm) & 5100 \\
Tangential force (N) & 177.48 \\
Axial force (N) & 0.3439 \\
Centrifugal (N) & 40,680 \\
\hline
\end{tabular}

\subsection{Modal Analysis}

Modal analysis was carried out on a turbine blade in other to determine the natural frequency and modes shape in every frequencies.

The gas turbine blade resonance frequency is given by equation 1;

$1 R P M=1 \div 60 \mathrm{~Hz}$

$5100 \mathrm{rpm}=(1 \div 60) \times 5100=85 \mathrm{~Hz}$

The basic equation solved in a typical undamped modal analysis is the classical eigenvalue problem;

$[K]\left\{\varnothing_{i}\right\}=\omega_{i}^{2}[M]\left\{\varnothing_{i}\right\}$

Where: [k] is the stiffness matrix, $\left\{\varnothing_{i}\right\}$ is the mode shape vector (eigenvector) of mode $i,[\mathrm{M}]$ is the mass matrix, $\omega$ is the natural frequency. Considering the time between amplitudes, the undamped $\left(\omega_{n r}\right)$ and damped $\left(\omega_{d r}\right)$ periods of vibration is given by equation 3 and 4 respectively [15];

$\omega_{n r}=\frac{2 \pi}{\tau_{d} \sqrt{1-\xi_{b z}^{2}}}$

$\omega_{d r}=\frac{2 \pi}{\tau_{d}}$

Where, $\tau_{d}$ is the damped period of vibration given by equation 5 ;

$\tau_{d}=t_{2}-t_{1}$

For structural analysis problem the displacement, stiffness and loads are related as given in equation 6 ; 
$[\mathrm{K}]\{\mathrm{X}\}=[\mathrm{F}]$

$\left[\begin{array}{ll}K 11 & K 12 \\ K 21 & K 22\end{array}\right]\left\{\begin{array}{l}X_{1} \\ X_{2}\end{array}\right\}=\left[\begin{array}{l}F_{1} \\ F_{2}\end{array}\right]$

Where, $[\mathrm{K}]=$ structural stiffness, $\{\mathrm{x}\}=$ Nodal displacement, $[\mathrm{F}]=$ load matrix, subscript 1 represents one physics, subscript 2 represents the other physics. Coupled effects are accounted for by the off-diagonal coefficient terms K11, K12, K21 and K22. By solving these equations at each node, we obtain the degrees of freedom, which would give the approximate behavior of complete model. The results of the solution are includes the nodal degree of freedom values, which form the primary solution, derived values, which form the element solution.

\section{Results and Discussion}

Modal analysis was carried out on a turbine blade in other to determine the natural frequency and modes shape in every frequencies. The natural frequency value of each part was depended on its geometry, dimension, material, elastic properties, mass and boundary condition. The blade was then designed in a manner, far from its resonance frequency. Thus reducing the amplitude of vibration and the risk of the part fracture. Results obtained from the modal analysis is presented in this section.

\subsection{Modal Analysis of IN 738 Blade Material}

Figure 3-8 represent the results obtained for modal analysis carried on IN 738 gas turbine blade material;

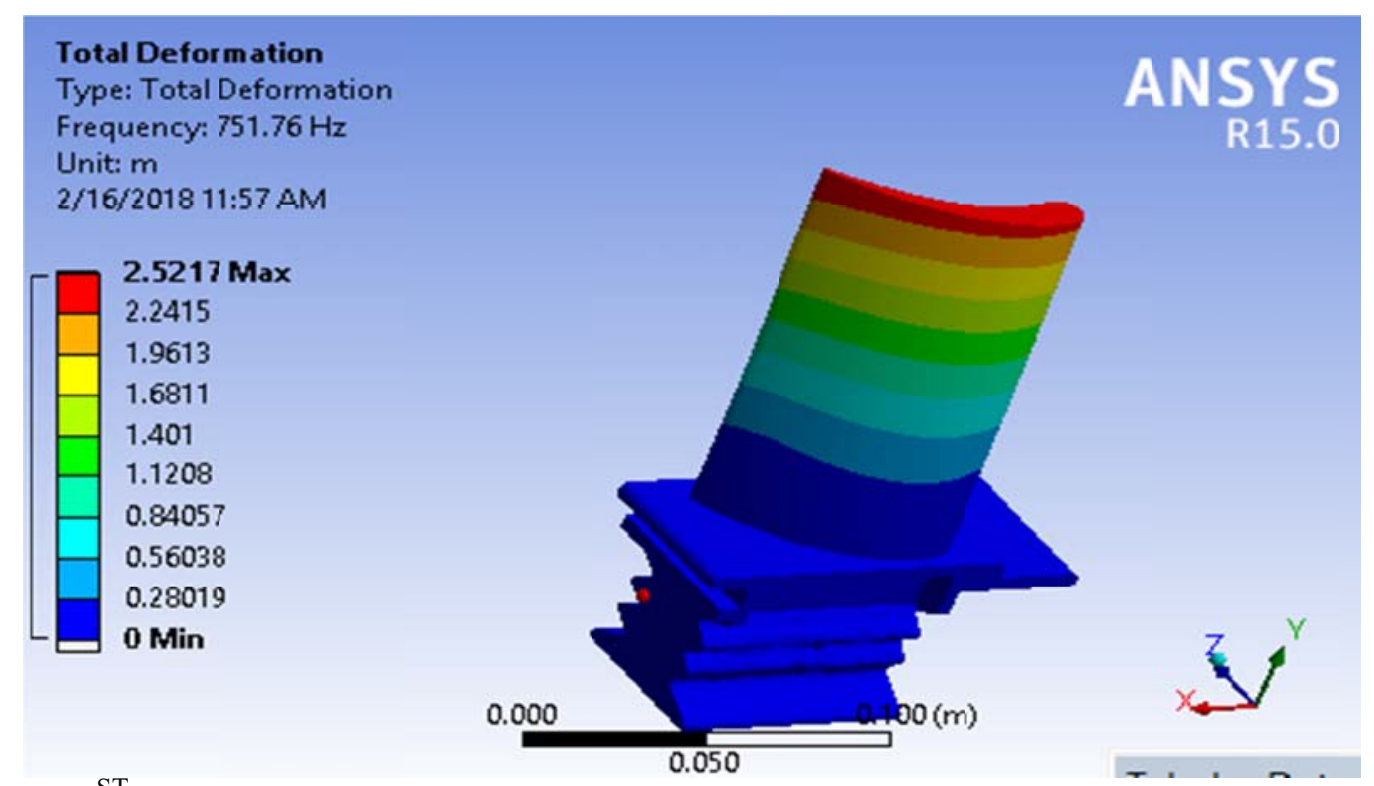

Figure 3. $1^{\text {ST }}$ Mode for IN 738 Blade 


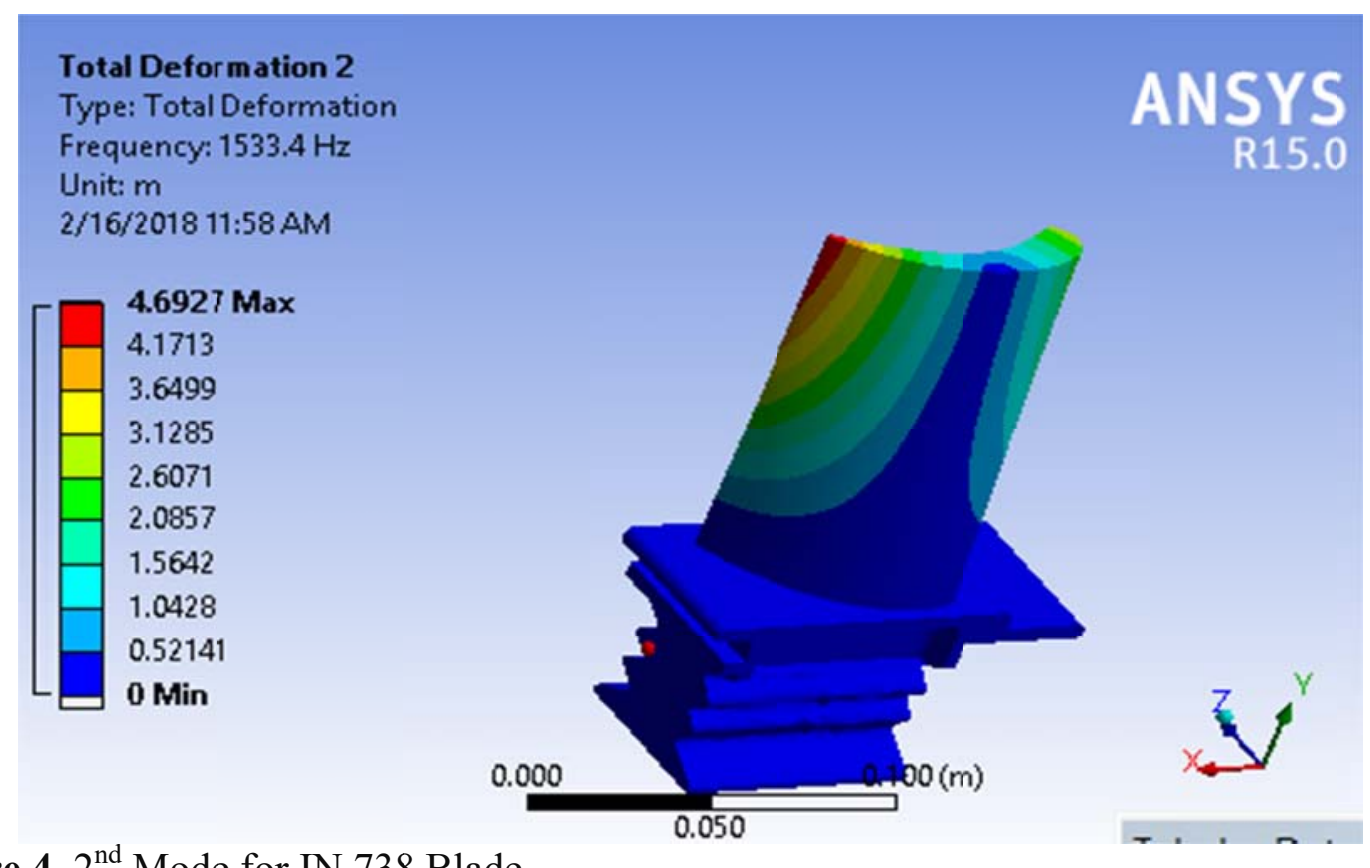

Figure 4. $2^{\text {nd }}$ Mode for IN 738 Blade

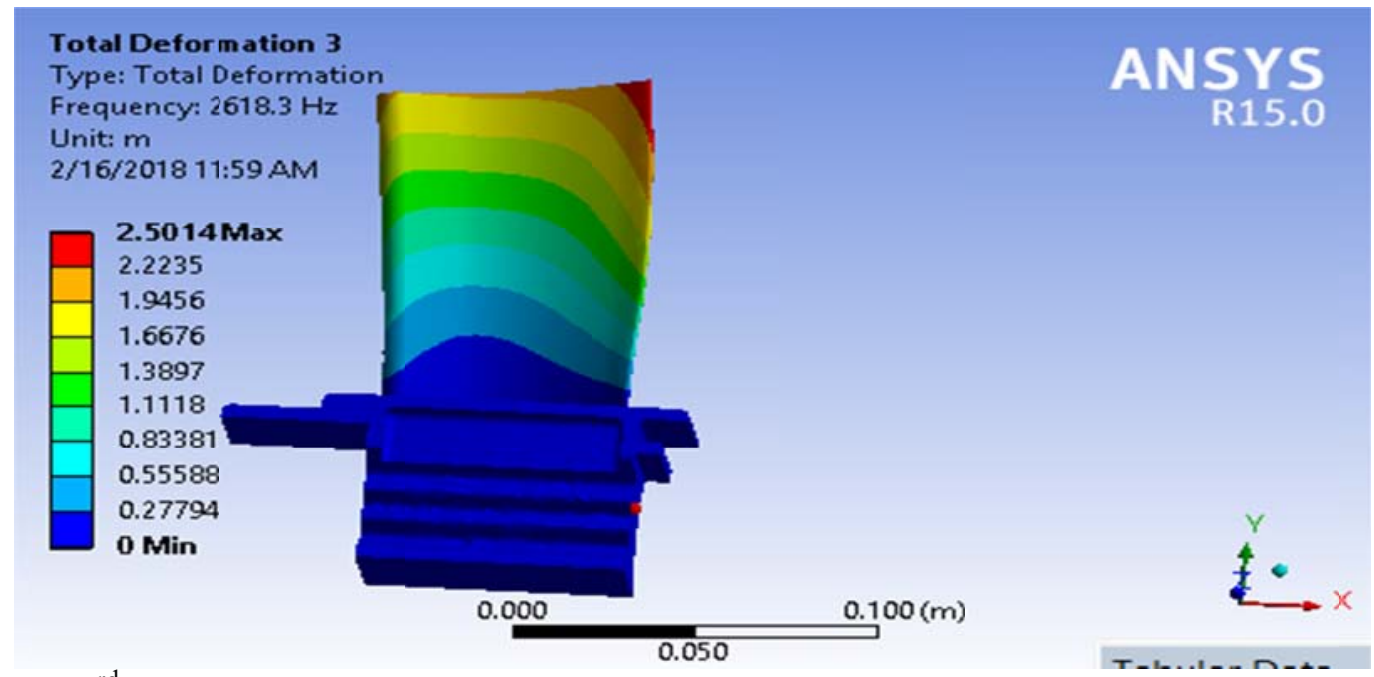

Figure 5. $3^{\text {rd }}$ Mode for IN 738 Blade 


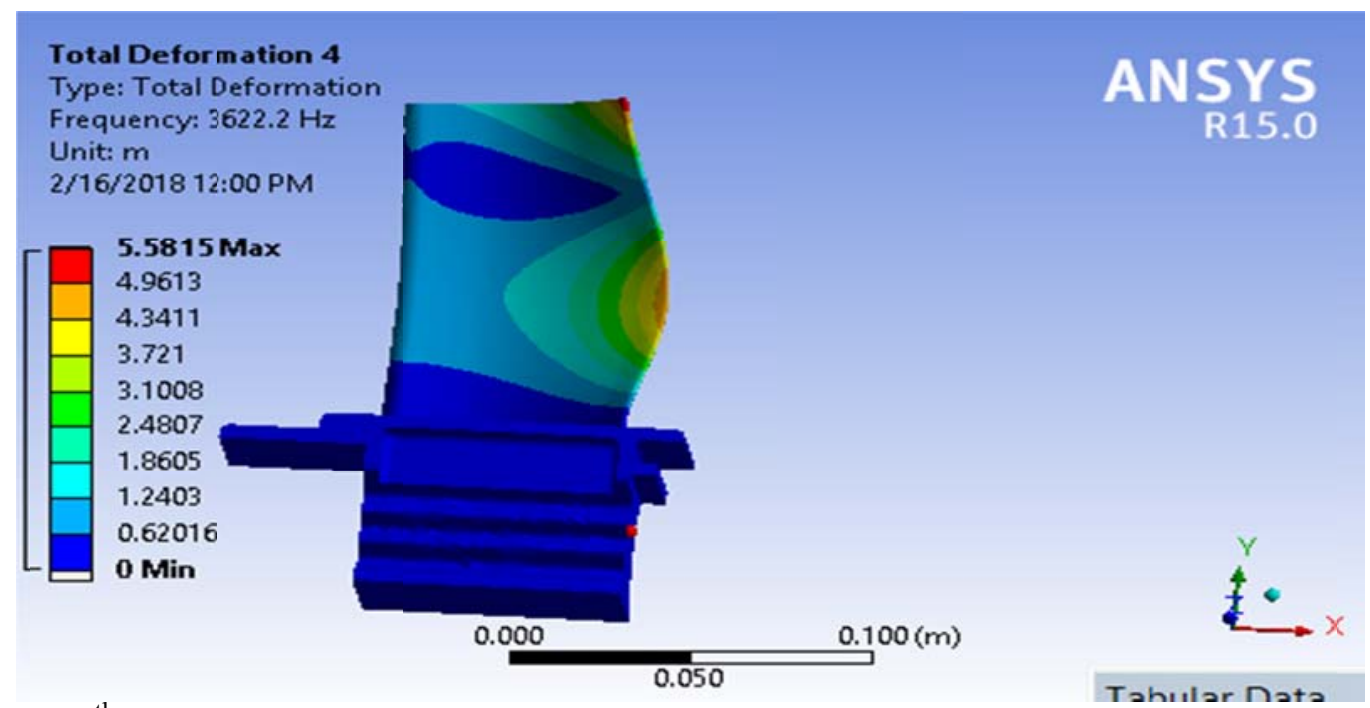

Figure 6. $4^{\text {th }}$ Mode for IN 738 Blade

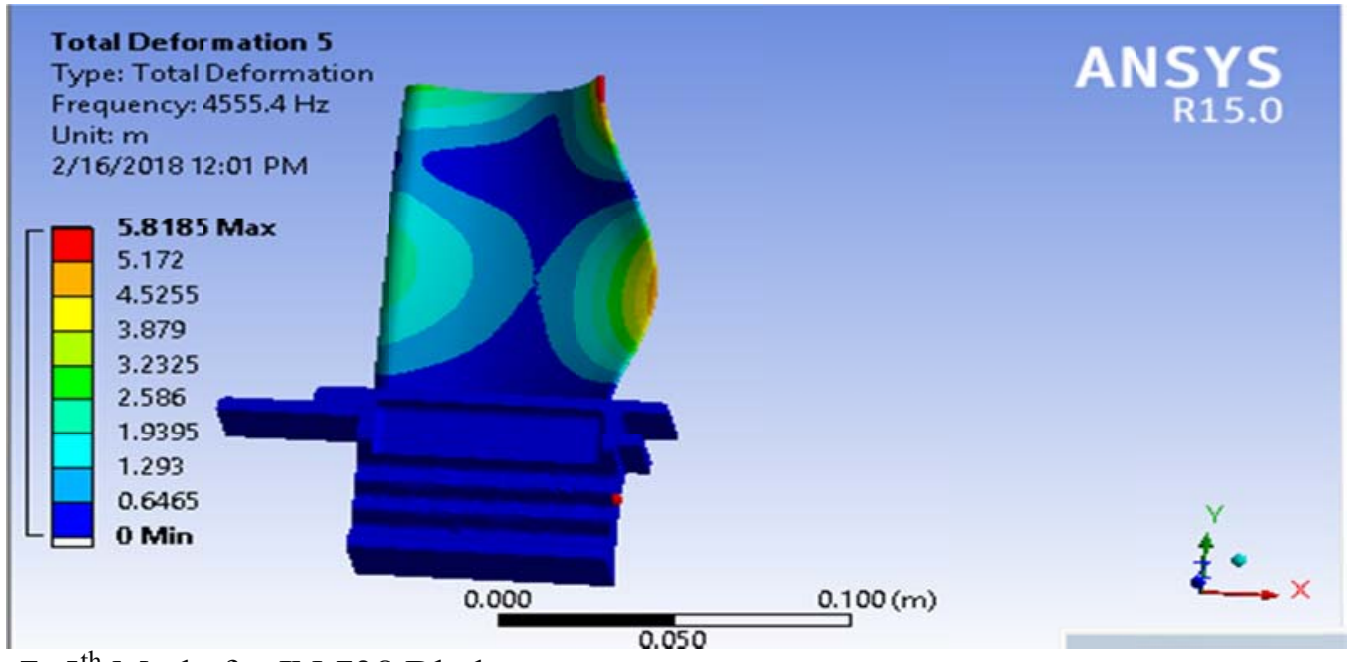

Figure 7. $5^{\text {th }}$ Mode for IN 738 Blade

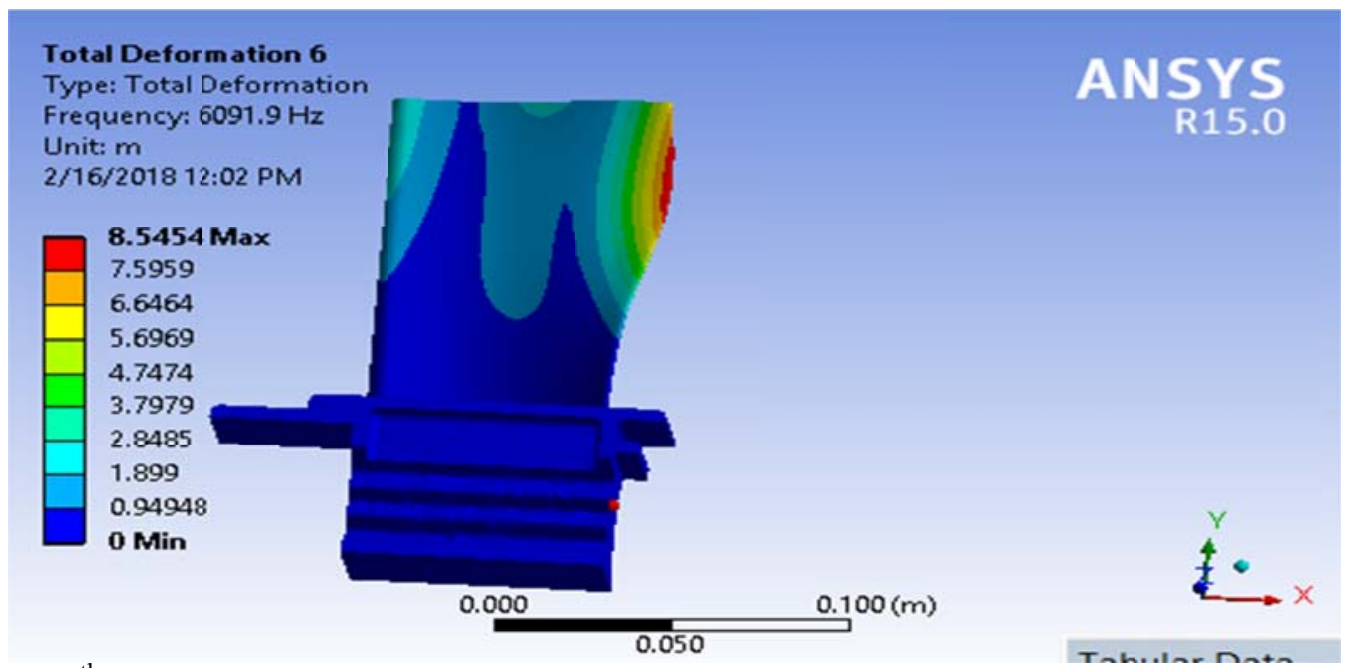

Figure 8. $6^{\text {th }}$ Mode for IN 738 Blade 
Figure 9 is a summary of the natural frequency in correspondence to the various mode shapes for IN 738 gas turbine blade material;

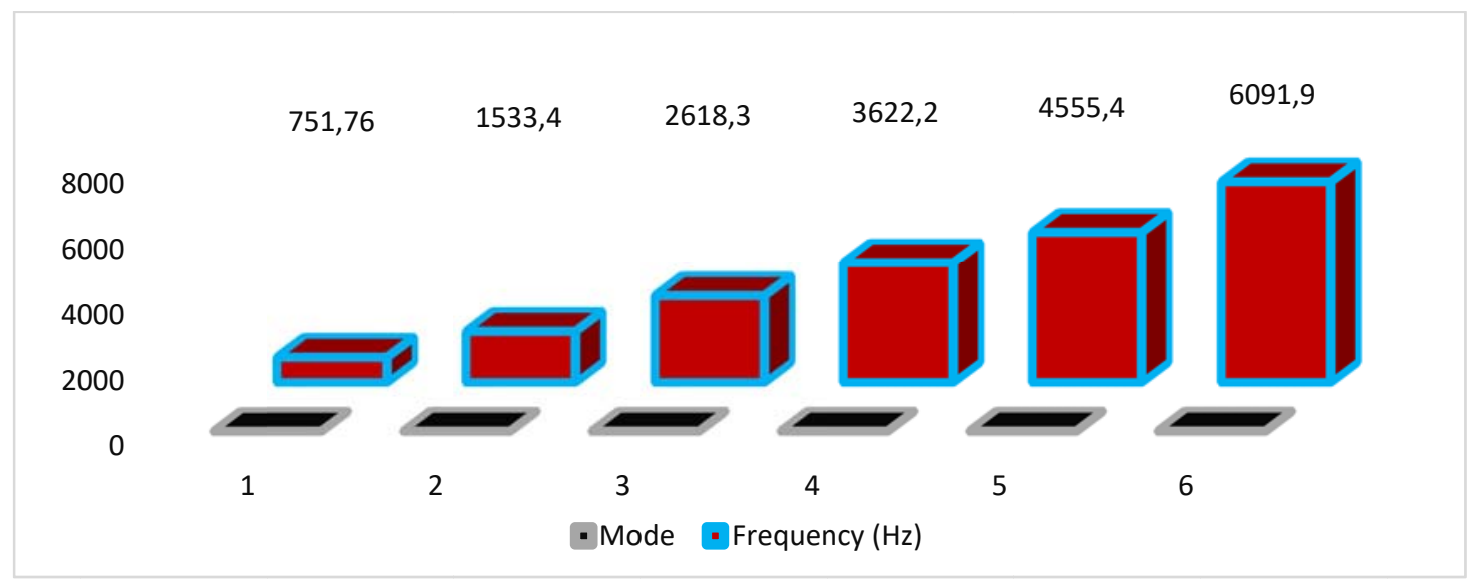

Figure 9. IN 738 Natural Frequencies

\subsection{Modal Analysis on U 500 Blade Material}

Figure 10-15 represent the results obtained for modal analysis carried on IN 738 gas turbine blade material;

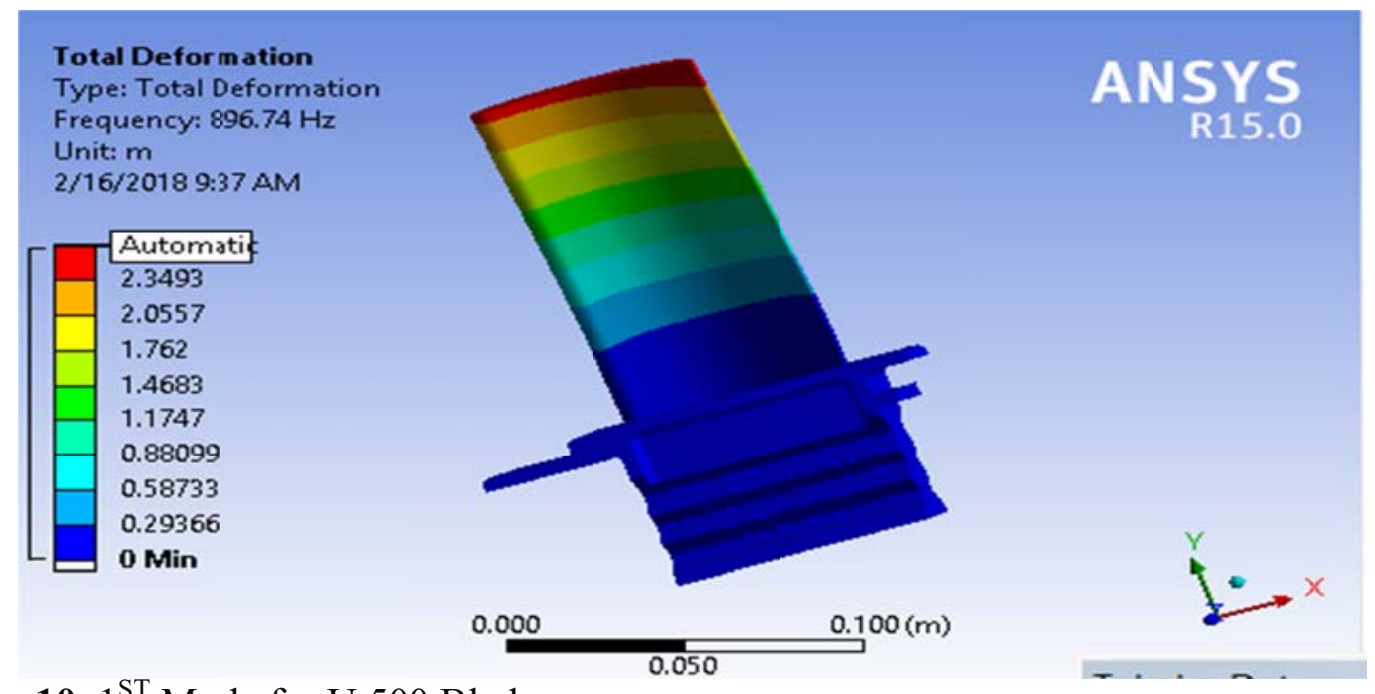

Figure 10. $1^{\mathrm{ST}}$ Mode for U 500 Blade 


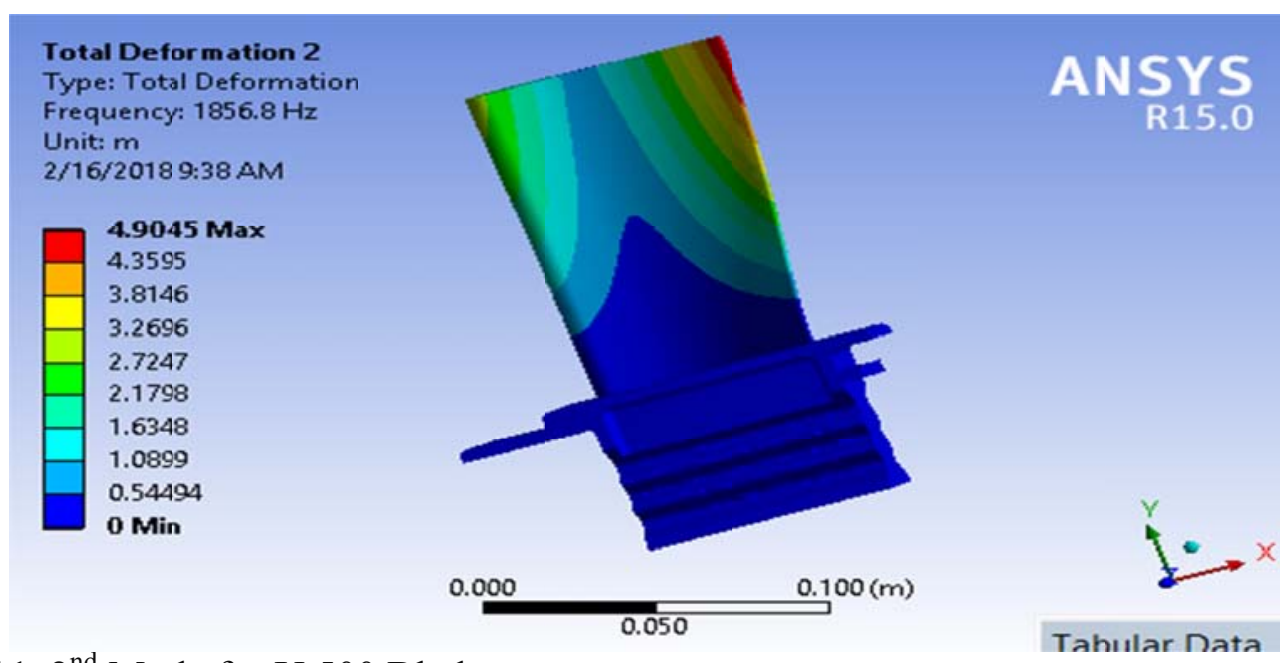

Figure 11. $2^{\text {nd }}$ Mode for U 500 Blade

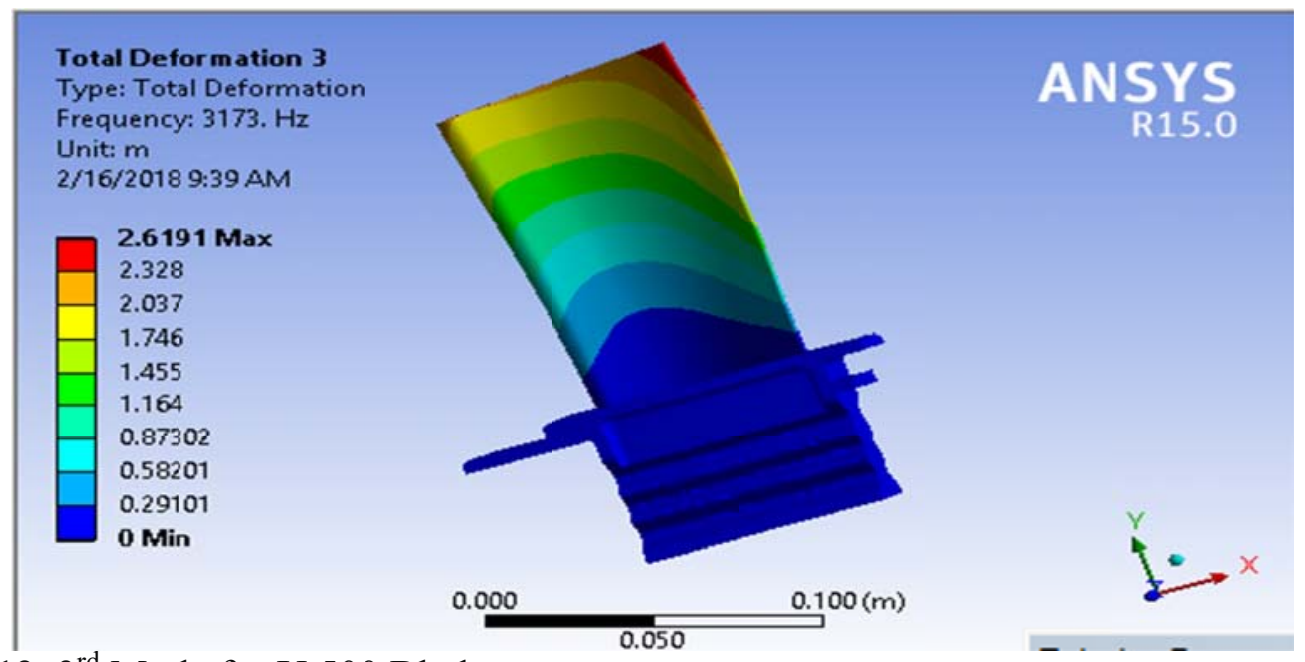

Figure 12. $3^{\text {rd }}$ Mode for U 500 Blade

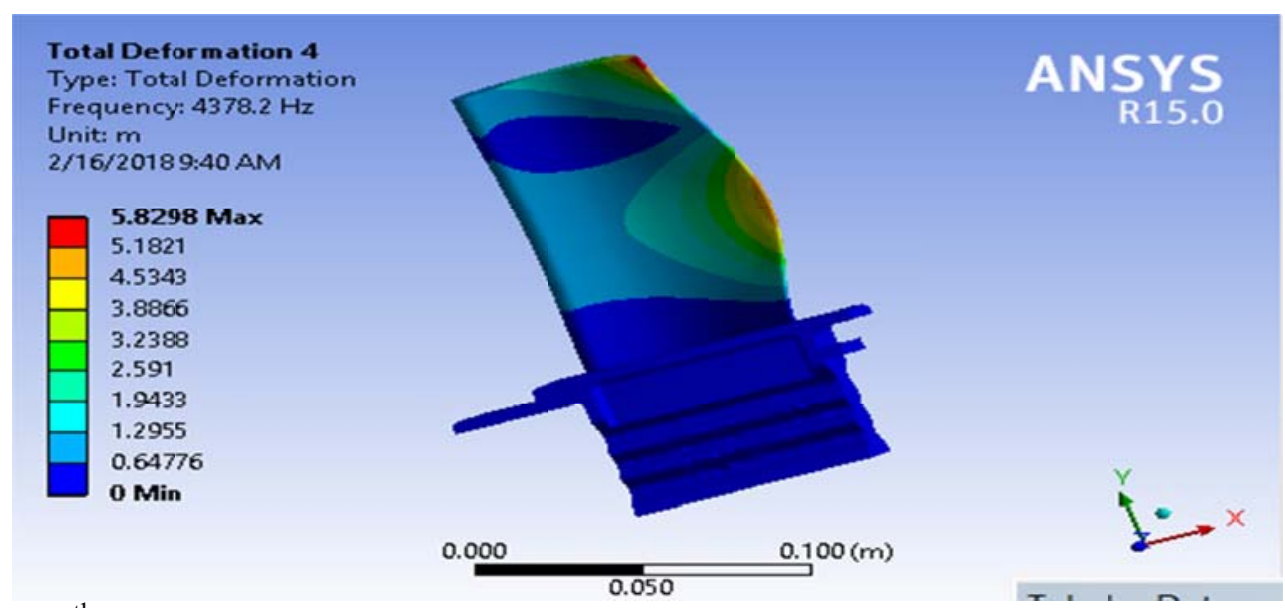

Figure 13. $4^{\text {th }}$ Mode for U 500 Blade 


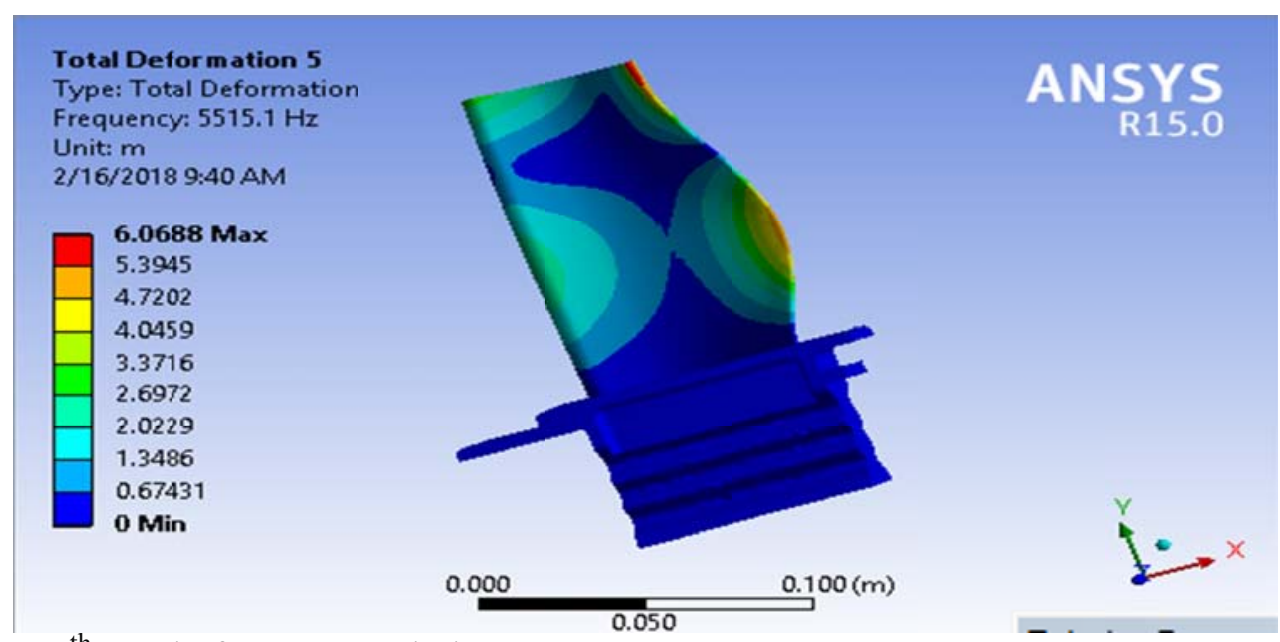

Figure 14. $5^{\text {th }}$ Mode for U 500 Blade

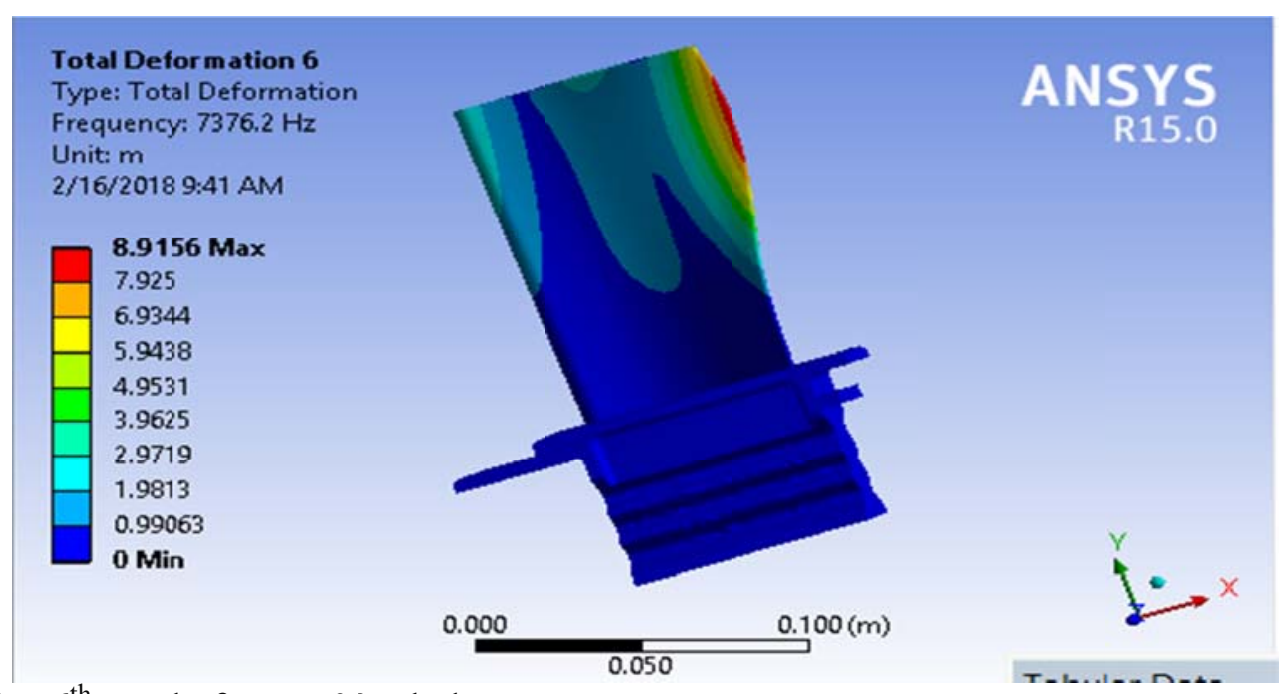

Figure 15. $6^{\text {th }}$ Mode for U 500 Blade

Figure 16 is a summary of the natural frequency in correspondence to the various mode shapes for U 500 blade material;

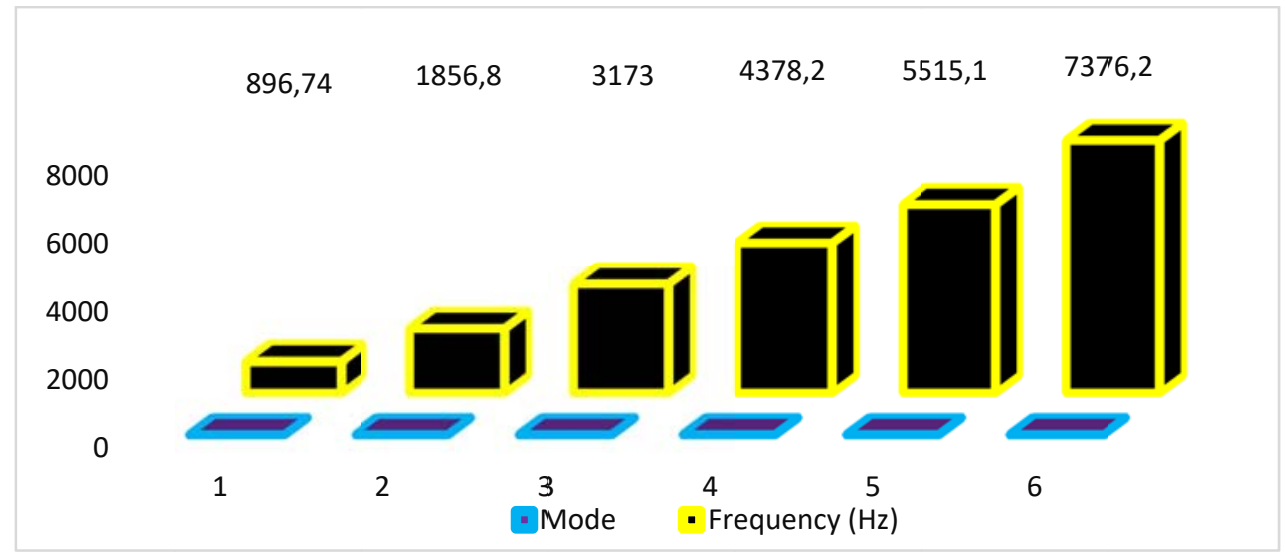

Figure 16. U 500 Natural Frequency 
Modal analysis was carried out on a turbine blade in other to determine the natural frequency and modes shape in every frequencies. The natural frequency value of each part was depended on its geometry, dimension, materials, elastic properties, mass and boundary conditions. The blade was then designed in a manner, different from its resonance frequency, thus, reducing the amplitude of vibration and the risk of fracture on the blade.

Natural frequency is the frequency at which an object vibrates when excited by a force. The structure possesses least resistance to the force and has a potential to fail if not controlled. Mode shape is deflection of object at a given natural frequency. Resonance is a condition where there is maximum amplitude of vibration and minimum resistance to oscillating force. For resonance condition to occur, the shape and frequency of a force must correspond to the natural frequency and mode shape of the structure (blade).

The boundary conditions for the modal analysis were linked with the boundary conditions from the static structural analysis. For this reason, all the stresses and deformations, which are created by the centrifugal force are included in the modal analysis. A rotating turbine blade has a larger bending stiffness than a stationary blade because rotation leads to stiffening effect due to the centrifugal force. The stiffening effects further depends on the rotational speed. For this reason, the natural frequency of a gas turbine blade is a function of the turbine rotational speed.

The mode shape contours are presented in the form of deformation in the three orthogonal direction. The color profile indicate areas of maximum deformation for the various modes, and also the corresponding natural frequencies for the mode shapes. There are several modes exhibited by the turbine blade which occur at various excitation frequencies such as first flexural or first flap (1F) mode (mode 1), first torsional mode (1T) by Mode 2, second flap mode (2F) by Mode 3, first edge bending mode (1E) by Mode 4, second torsional mode (2T) by Mode 5 and third Torsional Mode (3T) by mode 6. In the first mode, maximum deformation is at the tip and is minimal at the blade root. The second mode shows maximum deformation at the tip on the trailing edge which reduces towards the leading edge and minimum deformation at the root. In the third mode, deformation is all over the blade profile but reduces towards the root. In the fourth, fifth and sixth mode, maximum deformation is at the trailing edge but reduces towards the leading edge of the blade profile and is minimal at the blade root. However, investigation carried out by Ravi and Pandey [16] in similar area of study revealed maximum total deformation to occur at the tip portion of the blade profile.

The operational resonance frequency for the blade was $85 \mathrm{~Hz}$. The natural frequency across the two blade materials was far above the resonance frequency of the blade, and therefore dynamically stable. Furthermore, modal analysis was carried out to evaluate the dynamic stability of the rotor system. If the resonance is close to the operating speed range of the engine, it is possible to tune the frequencies away from the running range of the engine, but if the resonance is observed for many modes within the operating range of the engine, it is difficult to tune all the frequencies. The mode shape of the structure would then help in determining the potential failure modes that can be tuned to avoid the resonance. The results reveals that the fundamental frequency under the same load conditions were $751 \mathrm{~Hz}$ for IN 738 gas turbine blade material as shown in Figure 9 and $896 \mathrm{~Hz}$ for U500 gas turbine blade material as shown in Figure 16. This implies that the natural frequencies obtained are much higher than the operational frequency of $85 \mathrm{~Hz}$. Therefore, resonance is delayed across all blade materials, hence a given gas turbine system operating under the conditions employed in this study will be dynamically stable. 


\section{Conclusion}

From the above results, it was concluded that among the two turbine blade materials considered in this study, IN 738 is a better material for manufacturing of turbine blade of gas turbine engine in Trans-Amadi power plant. U500 blade material is generally used for the second stage rotor and would fail if used as a first stage blade material as examined in this study. IN738 is a suitable material for use as first stage rotor blade. This result serves as a guideline for the selection of material suitable for the manufacture of gas turbine blades. It also serves as a guide when conducting online monitoring and maintenance of the gas turbine in other to avoid unforeseen failure of turbine blades.

\section{Reference}

[1] Srinivasan, A. V., "Flutter and Resonant Vibration Characteristics of Engine Blades", International Gas Turbine and Aero engine Congress and Exhibition, Orlando, Florida, June 2-5, ASME Technical Paper No: 97-GT-533, (1997): 1-36.

[2] Sulaiman, K. S., Rameshkumar, G. R., "Vibration Diagnosis Approach for Industrial Gas Turbine and Failure Analysis", British Journal of Applied Science \& Technology, 14(2) (2016): 1-9.

[3] Barhm M., "Failure analysis of gas turbine blade using finite element analysis", International Journal of Mechanical Engineering and Technology, 7(3) (2017): 299-305.

[4] Ziegler, D., Puccinelli, M., Bergallo, B., Picasso, A., "Investigation of turbine blade failure in a thermal power plant", Case Studies in Engineering Failure Analysis, 1(3) (2013): 192-199.

[5] Ikpe, A. E., Owunna, I., Ebunilo, P. O., Ikpe, E., "Material Selection for High Pressure (HP) Compressor Blade of an Aircraft Engine", International Journal of Advanced Materials Research, 2(4) (2016a): 59-65.

[6] Ikpe, A. E., Owunna, I., Ebunilo, P. O., Ikpe, E., "Material Selection for High Pressure (HP) Turbine Blade of Conventional Turbojet Engines", American Journal of Mechanical and Industrial Engineering 1(1) (2016b): 1-9.

[7] Kalapala, P., Anjaneya, B. P., Anandarao, M., "Material Optimization and Dynamic Approach for performance criteria in application to Gas Turbine Blade to overcome resonance", International Journal of Scientific \& Engineering Research, 8(6) (2017): 189-196.

[8] Ivan B., Marco, A., Mathias, L., "Physically and Geometrically Non-linear Vibrations of Thin Rectangular Plates", International Journal of Non-Linear Mechanics 58 (2014): 30-40.

[9] Bhupendra, E. G., Sachin V. B., Kapil B. S., "Vibration Analysis of Gas Turbine Blade Profile Using Fem Technique and Tool", International Journal of Research in Advent Technology, 2(1) (2014): 182-189.

[10] Owunna, I., Ikpe, A. E., Satope, P., Ikpe, E., "Experimental Modal Analysis of a Flat Plate Subjected To Vibration", American Journal of Engineering Research (AJER), 5(6) (2016): 30-37. 
[11] Srinivasan, A. V., "Flutter and Resonant Vibration Characteristics of Engine Blades", Journal of Engineering for Gas Turbines and Power, 119(4) (1997): 742-775.

[12] Adawi, S. K., Rameshkumar, G. R., (2016) "Vibration Diagnosis Approach for Industrial Gas Turbine and Failure Analysis", British Journal of Applied Science and Technology, 14(2) (2016): 1-9.

[13] Lee C., "Recent Blade mounting Techniques", Journal of Engineering for Gas Turbines and Power, 89(3) (2015): 437-444.

[14] Sushila, R., Atul, K. A., Vikas, R. (2017) "Failure Analysis of a First Stage IN738 Gas Turbine Blade Tip Cracking in a Thermal Power Plant", Case Studies in Engineering Failure Analysis, 8 (2017): 1-10.

[15] Ikpe, A. E., Owunna, I., Satope, P., "Finite element analysis of aircraft tire behaviour under overloaded aircraft landing phase", Aeronautics and Aerospace Open Access Journal, 2(1) (2018): 34-39.

[16] Ravi, R. K., Pandey, K. M., "Static Structural and Modal Analysis of Gas Turbine Blade", IOP Conference Series: Materials Science and Engineering 225(012102) (2017): 1-9. 